\title{
Resonant Magnetotunneling via One-Dimensional Quantum Confined States
}

\author{
J. Wang,* P. H. Beton, N. Mori, ${ }^{\dagger}$ L. Eaves, H. Buhmann, \\ L. Mansouri, P. C. Main, T. J. Foster, and M. Henini \\ Department of Physics, University of Nottingham, Nottingham NG7 2RD United Kingdom
}

(Received 22 February 1994)

\begin{abstract}
Submicron resonant tunneling diodes fabricated by a novel processing technique exhibit two series of peaks in their current-voltage characteristics. The peaks are strongly dependent on both the magnitude and direction of a magnetic field oriented perpendicular to the tunnel current. This dependence is used to unambiguously identify resonant tunneling between one-dimensional quantum wire states.
\end{abstract}

PACS numbers: $73.40 . \mathrm{Gk}, 73.40 . \mathrm{Kp}, 73.50 . \mathrm{Jt}$

The current-voltage characteristics $[I(V)]$ of resonant tunneling diodes (RTDs) show pronounced resonant peaks due to electrons tunneling via the quasibound states formed within a quantum well. The original experiments on RTDs [1] used large area diodes in which electrons move as free particles in the plane of the quantum well. Following work by Reed et al. [2], several groups [36] have investigated the additional nonlinearities which are observed in the $I(V)$ of RTDs with submicron lateral dimensions and proposed that these features arise from quantization of the in-plane motion of electrons. In addition to lateral quantum confinement, additional peaks in $I(V)$ may arise from a completely different mechanismresonant tunneling via the localized bound states of shallow donors which are incorporated into the quantum well either unintentionally [7] or intentionally $[8,9]$. Thus it is clear that the appearance of additional peaks in the $I(V)$ of small area diodes cannot be taken in itself as conclusive evidence for lateral quantum confinement [10].

In this Letter we exploit a new processing technique to fabricate quantum wire RTDs with submicron lateral dimensions. The appearance of additional peaks in $I(V)$ due to lateral confinement is unambiguously demonstrated by the application of a high magnetic field.

The RTDs are fabricated from a GaAs/AlAs heterostructure grown by molecular beam epitaxy (MBE) on a semi-insulating GaAs(100) substrate. The active region consists of a GaAs quantum well (width $w=$ $9.0 \mathrm{~nm}$ ) formed between two AlAs tunnel barriers (thickness $b=4.7 \mathrm{~nm})$. The growth temperature $\left(550^{\circ} \mathrm{C}\right)$ and layer parameters were chosen to minimize the incorporation of unintentional donors in the quantum well [11] - in particular an undoped GaAs spacer layer (thickness $s=$ $20 \mathrm{~nm}$ ) was incorporated between each AlAs barrier and the adjacent doped GaAs contact layers. A schematic diagram of the device is shown in Fig. 1(a), with a definition of the $x, y, z$ axes. The active area of the device is the region of overlap of two GaAs bars, one (thickness $\ell_{t}$ ) etched in the top contact layer and the other (thickness $\ell_{b}$ ) in the lower contact layer. The AlAs barriers and GaAs quantum well are sandwiched in the region where the two bars overlap. The fabrication sequence is based
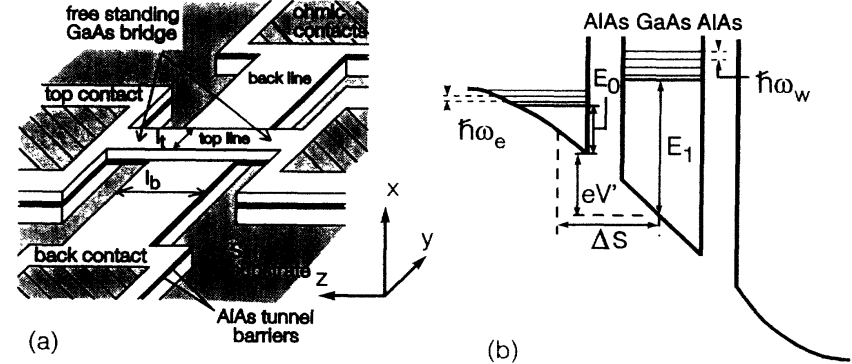

FIG. 1. (a) A schematic diagram of our device. (b) Conduction band profile of our device.

on a series of selective wet etches and is described fully in Ref. [12]. A notable feature of the device is the use of a freestanding GaAs bridge to provide a connection to a large Ohmic contact. The bridge resistance is of order $10 \mathrm{k} \Omega$ and varies by of order $10 \%$ over the field range discussed below. Optical lithography and undercut etching are used to achieve submicron dimensions. $\ell_{t}$ and $\ell_{b}$ can be $<0.5 \mu \mathrm{m}$, and typically $\ell_{t}<\ell_{b}$.

In Figure 2 we plot the low temperature $(T=0.3 \mathrm{~K})$ $I(V)$ for a device with $\ell_{t}=0.5 \mu \mathrm{m}$ and $\ell_{b}=1.0 \mu \mathrm{m}$. In forward bias electrons flow to the top contact. From a comparison of diodes with different dimensions (see Wang et al. [12]) we deduce that the sidewall depletion

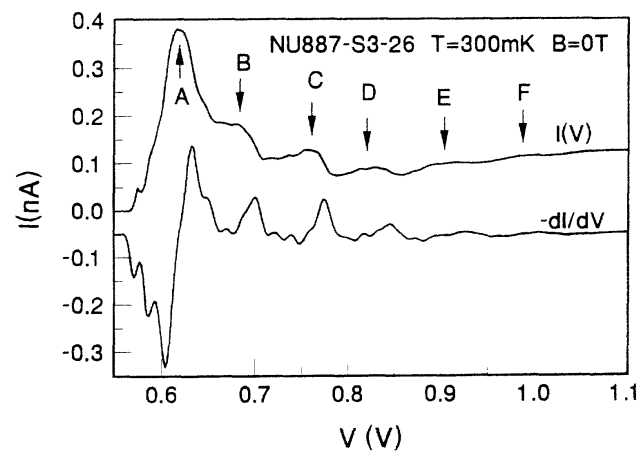

FIG. 2. Low temperature $(T=0.3 \mathrm{~K}) I(V)$ and $-d I / d V$ for a small area resonant tunneling diode. 
for these devices is $\sim 0.2 \mu \mathrm{m}$ for each edge and the current density is $J=18 \mathrm{nA} / \mu \mathrm{m}^{2}$. The dimensions of the active conducting region are therefore $\sim 100 \mathrm{~nm} \times$ $600 \mathrm{~nm}$ so that electrons tunnel between 1D quantum wires formed in the emitter and the quantum well.

Several clear peaks labeled $A-F$ are observed in the $I(V)$ shown in Fig. 2. The peak separation is $\sim 70 \mathrm{mV}$. In addition, there is a much weaker series of peaks with a separation of $\sim 20 \mathrm{mV}$. These peaks may be resolved more clearly in the differential conductance, $d I / d V$, also shown in Fig. 2. Similar peaks have been observed in many samples fabricated from several different $\mathrm{MBE}$ layers. The stronger series of peaks is still visible at $T=22 \mathrm{~K}$, but the weaker series is no longer resolved for $T>10 \mathrm{~K}$.

Figure 3 shows $I(V)$ for the same device in the presence of a magnetic field, $B$, oriented in the plane of the tunnel barriers, either perpendicular [Fig. 3(a)] or parallel [Fig. 3(b)] to the quantum wire. The curves in Figs. 3(a) and 3(b) display the same significant features after cycling to room temperature. We observe a clear anisotropy in the dependence of $I(V)$ on $B$ for the two field orientations. For $B$ perpendicular to the wire [Fig. 3(a)] peaks $A-F$ are shifted to higher voltage with increasing field. For a given field the shift in voltage is approximately the same for each of these peaks. As $B$ is increased beyond $\sim 3 \mathrm{~T}$ the peaks become broader and eventually cannot be resolved. In addition, for $B>4 \mathrm{~T}$ a weak series of features develops in the rise to the main resonance identified by arrows in Fig. 3(a) for the $B=10 \mathrm{~T}$ curve.

For $B \|$ wire [Fig. 3(b)] peaks $A-F$ show a complex splitting for small magnetic fields. However, for $B>3 \mathrm{~T}$ a regular series of peaks is observed in $I(V)$ with a typical spacing of $\sim 30 \mathrm{mV}$. As $B$ is increased a given peak first increases in amplitude and shifts to lower voltage, and then for a further increase in field it decreases in amplitude and

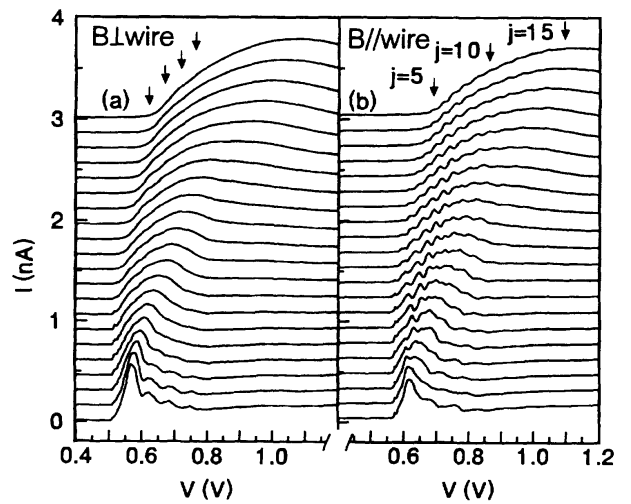

FIG. 3. $I(V)$ in the presence of a magnetic field between $B=0 \mathrm{~T}$ (lowest curve) and $B=10 \mathrm{~T}$ (top curve) in $0.5 \mathrm{~T}$ steps. The field is oriented perpendicular to the current flow and either perpendicular (a) or parallel (b) to the axis of the quantum wire. finally disappears. Remarkably we are able to observe more than 15 peaks in this series as labeled in Fig. 3(b).

This anisotropic dependence on $B$ provides strong empirical evidence that the additional peaks in $I(V)$ are related to the shape of the device, and are therefore due to lateral quantum confinement rather than inhomogeneities. Classically the field gives rise to a Lorentz force. The anisotropy occurs since this force may be parallel or perpendicular to the wire leading respectively to a strong or weak interplay with the confining potential. In a corresponding quantum mechanical formulation the current is determined by the matrix element between initial and final confined states which is strongly dependent on a parallel field, but independent of a perpendicular field as we show below.

To explain our data we have developed a simple model for resonant tunneling through laterally bound states. For a large area diode the confinement energy of the lowest quasibound state in the quantum well is $E_{1}(\sim 40 \mathrm{meV}$ for our heterostructure), and the bound state energy of the two-dimensional accumulation layer formed under bias at the emitter/barrier interface is $E_{0}$ [see Fig. 1(b)]. For our small area diode we assume that the well and emitter states are laterally quantized in the $y$ direction by a parabolic potential with energy levels spaced by $\hbar \omega_{w}$ and $\hbar \omega_{e}$, respectively. [See schematic conduction band diagram in Fig. 1(b).] From our previous work [13] on small area RTDs we expect the degree of confinement to be different in the emitter and the well, and that in forward bias $\hbar \omega_{w}>\hbar \omega_{e}$. Free electron motion is assumed for the $z$ direction. Thus a set of 1D subbands is formed in the emitter (denoted by index $i$ ) and well (index $j$ ).

The current is calculated from the transfer Hamiltonian formalism. Our theory is described in more detail in Ref. [14]. See also Demmerle et al. [15] for a treatment of a related low-dimensional tunneling problem and Bryant [16]. Figures 4(a) and 4(b) show the calculated current as a function of $V^{\prime}\left[V^{\prime}\right.$ is the voltage dropped between the emitter/tunnel barrier interface and the center of the quantum well-see Fig. 1(b)] in the presence of a magnetic field perpendicular [Fig. 4(a)] or parallel [Fig. 4(b)] to the wire axis. The following parameters were used: $\hbar \omega_{w}=3.5 \mathrm{meV}, \hbar \omega_{e}=1.5 \mathrm{meV}$, $E_{F}=6 \mathrm{meV}$, and $\Gamma=1.5 \mathrm{meV}$. The value of $E_{F}$, the Fermi energy in the emitter, is extracted from magnetooscillations in the tunnel current of a large area RTD [17]. $\Gamma$ is a parameter characterizing the width of the energy levels. The choice of the other parameters is justified below. There is remarkable agreement between the calculated and experimental curves. In particular, the calculated curves show weak and strong peaks with approximately the correct relative magnitudes with a magnetic field dependence that corresponds closely to experiment for each field orientation.

For $B \|$ wire the resonant current between states $i$ and $j$ is $I_{i j} \propto\left|M_{i j}\right|^{2} k_{F}^{i}$ where $k_{F}^{i} \quad\left(=\left\{2 m\left[E_{F}-(i+\right.\right.\right.$ 


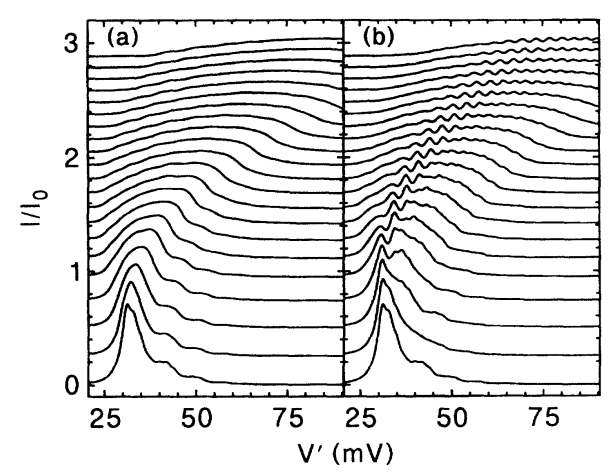

FIG. 4. Calculated plots of $I(V)$ for comparison with experimental data in Fig. 3. The calculated curves are for fields between $B=0 \mathrm{~T}$ (lowest curve) and $B=10 \mathrm{~T}$ (top curve) in $0.5 \mathrm{~T}$ steps. (b) is for a magnetic field oriented perpendicular (parallel) to the axis of the quantum wire. Parameters as discussed in text. $I_{0}$ is an arbitrary constant.

$\left.\left.1 / 2) \hbar \omega_{e}\right]\right\}^{1 / 2} / \hbar$ ) is the Fermi wave vector of the $i$ th 1D subband and $M_{i j}$ is the magnetic-field-dependent matrix element given by

$$
\begin{aligned}
M_{i j} & =\int_{-\infty}^{\infty} e^{-i k_{0} y} \phi_{i}(y) \phi_{j}(y) d y \\
& =\int_{-\infty}^{\infty} \Phi_{i}\left(k_{y}-k_{0}\right) \Phi_{j}\left(k_{y}\right) d k_{y} .
\end{aligned}
$$

$\phi_{i}(y)$ and $\phi_{j}(y)$ are the $(B=0 \mathrm{~T})$ wave functions of the initial and final states and $\Phi_{i}\left(k_{y}\right)$ and $\Phi_{j}\left(k_{y}\right)$ are their Fourier transforms. $k_{0}=e B \Delta s / \hbar$ is a magnetic-fielddependent wave vector shift $[\Delta s$ is the spatial separation of the initial and final states in the $x$ direction; see Fig. 1(b)]. Note that for the special case of large area diodes $\phi_{i}(y)$ and $\phi_{j}(y)$ are plane waves, and $k_{0}$ enters as a wave vector shift of the initial state [18].

Figure 5 shows the theoretical $I(V)$ for $B=0 \mathrm{~T}$ and $B=6 \mathrm{~T}$ for $\Gamma=1.5 \mathrm{meV}$. The calculated contributions to the current, $I_{i j}$, from individual transitions between state $i$ in the emitter and state $j$ in the well are also shown and represented by the vertical lines in Fig. 5. These lines would correspond to $I(V)$ in the limit of zero broadening, $\Gamma=0$. The transitions are classified by the pair of integers $(i, j)$ (see Fig. 5). For $B=0 \mathrm{~T}$ the peak corresponding to peak $A$ in our data is composed of transitions between states with the same index, i.e., $(0,0),(1,1),(2,2)$, and $(3,3)$. We show below that there are only four levels below the Fermi level in the emitter so we do not see any transitions for $i>3$. The voltage $\left(V^{\prime}\right)$ separation of these transitions is $\Delta V^{\prime}=\hbar \omega_{w}-\hbar \omega_{e}$. The next sequence of transitions which occur is $(0,2)$, $(1,3),(2,4)$, and $(3,5)$, in which the change of index $j-i=2$, followed by sequences for which $j-i=$ $4,6, \ldots$. The effect of broadening is to merge these sets of transitions (see curve for $\Gamma=1.5 \mathrm{meV}$ ) so that the peaks that are most clearly resolved arise from the sets

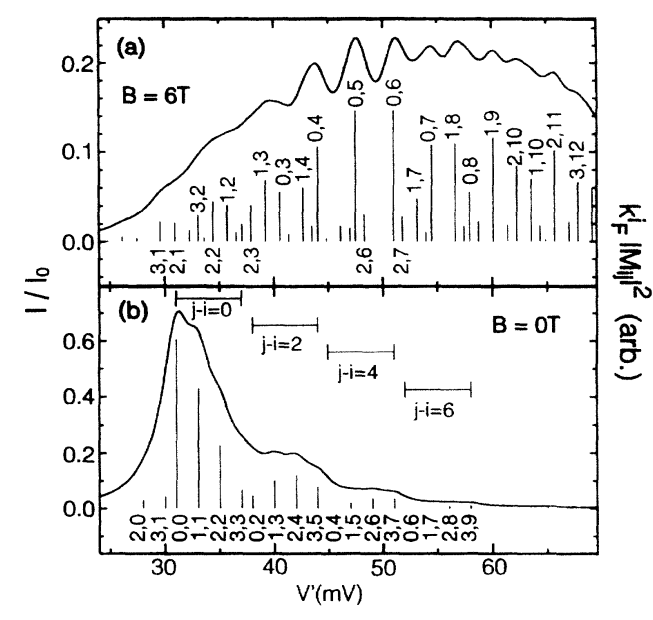

FIG. 5. Theoretical curves for $B=0 \mathrm{~T}$ and $B=6 \mathrm{~T}$ replotted together with the quantity $\left|\boldsymbol{M}_{i j}\right|^{2} k_{F}^{i}$, which is proportional to the current flowing between a particular pair of states, $I_{i j}$, denoted $(i, j)$. For $B=0 \mathrm{~T}$ the strongest peaks comprise the sets of transitions $j-i=0,2,4,6, \ldots$ and have a period in voltage $\propto 2 \hbar \omega_{w}$. The weaker structure has a voltage period $\propto\left(\hbar \omega_{w}-\hbar \omega_{e}\right)$. At high field parity conservation is broken and the voltage period $\propto \hbar \omega_{w}$.

of transitions $j-i=0,2,4,6, \ldots$ corresponding to peaks $A, B, C, D, \ldots$ in our data. The voltage $\left(V^{\prime}\right)$ separation of these peaks is $\sim 2 \hbar \omega_{w}$. Our calculations show that we expect to resolve these peaks clearly only for $E_{F}<2 \hbar \omega_{w}$.

Note that for $B=0 \mathrm{~T}$ we do not observe transitions between states with different symmetry which would correspond to $j-i=$ odd integer, since $M_{i j}=0$ for $j-i=$ odd. However, for $B>0 \mathrm{~T}$ this parity conservation may be broken. This may be seen from Fig. 5(a), which shows a series of peaks due to tunneling between states with different symmetry. At high field after taking account of broadening there is only one peak in $I(V)$ due to tunneling via a particular laterally quantized state in the well, and the voltage $\left(V^{\prime}\right)$ period is therefore given by $\hbar \omega_{w}$. This is implied by the labeling $j=0-15$ in Fig. 3(b).

The ratio of the voltage periods at $B=0 \mathrm{~T}$ and high field should therefore be close to 2 , in excellent agreement with our experimental data (voltage periods are given by 70 and $30 \mathrm{mV}$ ). However, it is also possible to deduce an absolute value for $\hbar \omega_{w}$ as follows. A high energy simple harmonic oscillator state has a pronounced extremal maximum in $\left|\Phi_{j}\right|^{2}$ at a value $k_{j, \max }(= \pm[2(j+$ $\left.\left.1 / 2) \hbar \omega_{w}\right]^{1 / 2} / \hbar\right)$ [19]. Our theory shows that the strongest peak in $I(V)$ occurs for the state in the well for which $k_{0}=k_{j, \max }$. For example, for $B=6 \mathrm{~T}$ our experimental data show that the strongest peak comes from tunneling via the $j=5$ state. Using a value $\Delta s=20 \mathrm{~nm}$ this gives $\hbar \omega_{w}=3.5 \mathrm{meV}$, the value used in our calculations. From our high field data we deduce the leverage ratio between $V^{\prime}$ and the total applied voltage $V$ is $k \sim 8.5$. This is slightly higher than the value deduced for a large 
area diode $(k=6)$. It is likely that this difference is caused by the one-dimensional nature of the depletion region set up under bias. Note also that for a small area diode the voltage position of the main resonance (peak A) occurs at a higher voltage than for a large area diode. This is due to an electrostatic effect as discussed by Reed et al. [20].

We are also able to deduce $\hbar \omega_{e}=1.5 \mathrm{meV}$. Confirmation of this value comes from the effect of field for $B$ perpendicular to the wire, $B \| y$. The weak structure we observe in our data at high field [see arrows in Fig. 3(a)] is a direct consequence of the quantization of states in the emitter. We deduce that there are four 1D subbands below the Fermi energy, $E_{F} / \hbar \omega_{e} \sim 4$, the value used in our calculations. Note that $M_{i j}$ is not a function of field for this orientation, so the peaks $A-F$ are all expected to have approximately the same dependence on $B$ in accordance with our experimental data.

The value of $\Gamma=1.5 \mathrm{meV}$ is much larger than the expected intrinsic energy width $(\sim 1 \mu \mathrm{eV})$ of the states in the quantum well. We believe the principal contribution to $\Gamma$ comes from the inhomogeneous broadening caused by fluctuations of the conduction band minimum along the length of our quantum wire. Finally, a sharp rise in current is observed below peak $A$ at the voltage threshold for current flow. This is not predicted by our theory, nor does it display a clear anisotropic dependence on $B$. It is possible that this feature is a Fermi edge singularity similar to that which we have recently reported [21].

In conclusion, we have shown that peaks in $I(V)$ due to lateral quantum confinement may be unambiguously identified from their dependence on a magnetic field oriented perpendicular to the current. At high magnetic field we are able to resolve equally spaced peaks in $I(V)$ from tunneling via the lowest 15 states, confirming that the confining potential for our device is parabolic.

This work was funded by the U.K. Science and Engineering Council and by the EC Basic Research Action No. 7193 (PARTNERS). P.H.B. and L.E. are grateful to the Royal Society, and N.M. to the British Council for financial support.

*Present address: Department of Physics, HKUST, Clear Water Bay, Kowloon, Hong Kong.

†'Permanent address: Department of Electronic Engineering, Osaka University, Osaka, Japan.

[1] L. L. Chang, L. Esaki, and R. Tsu, Appl. Phys. Lett. 24, 593 (1974).

[2] M. A. Reed, J.N. Randall, R. J. Aggarwal, R. J. Matyi, T. M. Moore, and A.E. Wetsel, Phys. Rev. Lett. 60, 535 (1988).
[3] M. Tewordt, V.J. Law, M. J. Kelly, R. Newbury, M. Pepper, D. C. Peacock, and J.E. F. Frost, J. Phys. Condens. Matter 2, 8969 (1990).

[4] P. Gueret, N. Blanc, R. Germann, and H. Rothuizen, Phys. Rev. Lett. 68, 1896 (1992).

[5] S. Tarucha, Y. Hiarayama, T. Saku, and T. Kimura, Phys. Rev. B 41, 5459 (1990).

[6] B. Su, V.J. Goldman, M. Santos, and J. E. Cunningham, Appl. Phys. Lett. 58, 747 (1991).

[7] M.W. Dellow, P.H. Beton, C. J.G. M. Langerak, T.J. Foster, P. C. Main, L. Eaves, M. Henini, S. P. Beaumont, and C. D. W. Wilkinson, Phys. Rev. Lett. 68, 1754 (1992).

[8] J-W. Sakai, T.M. Fromhold, P. H. Beton, L. Eaves, M. Henini, P. C. Main, F. W. Sheard, and G. Hill, Phys. Rev. B 48, 5664 (1993).

[9] J-W. Sakai, N. La Scala, Jr., P. C. Main, P. H. Beton, T. J. Foster, A. K. Geim, L. Eaves, M. Henini, G. Hill, and M. A. Pate, Solid State Electron. 37, 965 (1994); see also M. Tewordt et al., Solid State Electron. 37, 793 (1994).

[10] P. H. Beton, L. Eaves, and P. C. Main, Phys. Rev. Lett. 69, 2995 (1992).

[11] M. Henini, J-W. Sakai, P. H. Beton, L. Eaves, P. C. Main, and G. Hill, J. Vac. Sci. Technol. B 11, 958 (1993); see also S. Muto et al., Jpn. J. Appl. Phys. 25, L577, for a discussion of the influence of spacer layers on peak to valley ratios.

[12] J. Wang, P. H. Beton, H. Buhmann, L. Mansouri, L. Eaves, P. C. Main, T. J. Foster, and M. Henini, Appl. Phys. Lett. (to be published).

[13] P.H. Beton, M.W. Dellow, P.C. Main, L. Eaves, M. Henini, T. J. Foster, S.P. Beaumont, and C.D.W. Wilkinson, Appl. Phys. Lett. 60, 2508 (1992).

[14] N. Mori, P.H. Beton, J. Wang, and L. Eaves, (to be published).

[15] W. Demmerle, J. Smoliner, E. Gornik, G. Bohm, and G. Weimann, Phys. Rev. B 47, 13574 (1993).

[16] G. W. Bryant, Phys. Rev. B 39, 3145 (1989).

[17] V. J. Goldman, D.C. Tsui, and J.E. Cunningham, Phys. Rev. B 35, 9387 (1987).

[18] M. L. Leadbeater, L. Eaves, P.E. Simmonds, G. A. Toombs, F. W. Sheard, P. A. Claxton, G. Hill, and M. A. Pate, Solid State Electron. 31, 707 (1988); see also J. Smoliner, W. Demmerle, G. Berthold, E. Gornik, G. Weimann, and W. Schlapp, Phys. Rev. Lett. 63, 2116 (1989).

[19] L.I. Schiff, Quantum Mechanics (McGraw-Hill, New York, 1949), p. 66.

[20] M.A. Reed, J.H. Luscombe, J.N. Randall, W.R. Frensley, R.J. Aggarwal, R.J. Matyi, T.M. Moore, and A.E. Wetsel, Science and Engineering of 1 and 0 Dimensional Semiconductors, NATO ASI Ser. B, Vol. 214 (Plenum, New York, 1990).

[21] A. K. Geim, P. C. Main, N. La Scala, Jr., L. Eaves, T. J. Foster, P. H. Beton, J-W. Sakai, F. W. Sheard, M. Henini, G. Hill, and M. A. Pate, Phys. Rev. Lett. 72, 2061 (1994). 


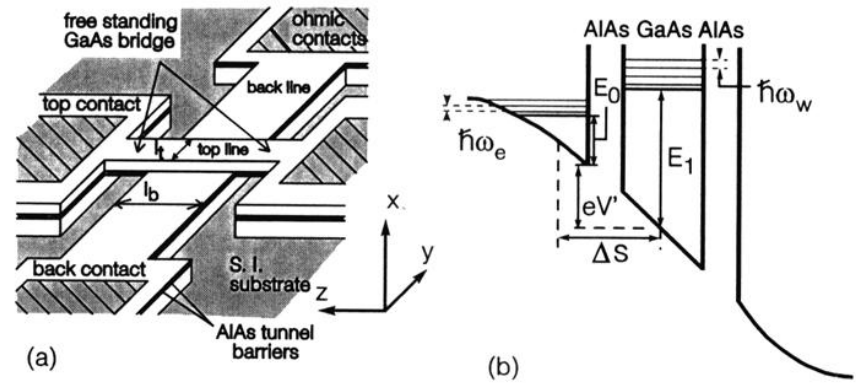

FIG. 1. (a) A schematic diagram of our device. (b) Conduction band profile of our device. 